Pacific Journal of Mathematics

TORUS GROUP ACTIONS ON SIMPLY CONNECTED 


\title{
TORUS GROUP ACTIONS ON SIMPLY CONNECTED MANIFOLDS
}

\author{
Soon-Kyu Kim, Dennis McGavran, Jingyal PaK
}

In this paper it is shown that effective torus $T^{n}$-actions on simply connected closed $(n+2)$-manifolds $M^{n+2}$ for all $n \geqq 1$ exist, and a complete orbit structure is given. It turns out that all maximal isotropy subgroups must generate the whole group $T^{n}$. The cross-sectioning theorem for the orbit map $\pi: M \rightarrow M^{*}=M^{n+2} / T^{n}$ is given, and as its application an equivariant classification theorem is obtained.

It is also shown that free torus $T^{n}$-actions on simply connected closed $(n+4)$-manifolds for all $n \geqq 1$ exist.

The purpose of this paper is to study the actions of $T^{n}$ on simply connected manifolds with low codimension. It is shown here that the orbit space $M^{*}=M^{n+2} / T^{n}$ is a disk $D^{2}$ with the boundary points corresponding to only isotropy subgroups $T^{1}$ 's and $T^{2}$ 's of $T^{n}$ and the interior points corresponding to only principal orbits.

It is also given that a subgroup $T^{k}(n>k \geqq 1)$ cannot contain all nonfree elements of $T^{n}$, where, by a nonfree element we mean an element that fixes some points of $M^{n+2}$. This implies that all maximal isotropy subgroups (which are $T^{2}$ 's) must generate the whole group $T^{n}$.

We prove that if $T^{n}$ acts on a closed orientable $(n+2)$-manifold $M^{n+2}$ with one or two orbit types of orbit structure, and with simply connected orbit space then $M^{n+2}$ is homeomorphic to $L(p, q) \times T^{n-1}$ for $n \geqq 2$. Hence $T^{n}$ cannot act freely on any simply connected closed $(n+2)$-manifold $M^{n+2}$ for $n>1$. However, we demonstrate here the existence of a free $T^{n-2}$-actions on a simply connected closed $(n+2)$ manifold.

It is known that $T^{n}$ cannot act effectively on a simply connected closed $(n+1)$-manifold $M^{n+1}$ for $n \geqq 3$ [4], and with the above statements we suspected that $T^{n}$ cannot act effectively on a simply connected $(n+2)$-manifold $M^{n+2}$ for $n \geqq 5$. However, we prove the existence theorem of $T^{n}$-actions on simply connected closed $(n+2)$ manifold $M^{n+2}$ for all $n$.

We state the cross-sectioning theorem and equivariant classification theorem without proof.

As a result, we more or less know completely about torus group actions on simply connected closed manifolds with the low codimension. That is, $T^{n}$ cannot act freely on a simply connected closed $(n+1)$ or $(n+2)$-manifold, but $T^{n}$ can act freely on a simply connected closed $(n+4)$-manifold; and $T^{n}$ cannot act effectively on a simply 
connected closed $(n+1)$-manifold for $n \geqq 3$, but $T^{n}$ can act effectively on a simply connected closed $(n+2)$-manifold.

Although most of our results carry over to the topological category we will work in the locally smooth category. Definitions and terminologies are all standard and can be found in Bredon's book [1].

1. Isotropy subgroups and applications. Let a torus group $T^{n}$ act effectievely on a simply connected closed $(n+2)$-manifold $M^{n+2}$. Then by the slice theorem [2] there are principal orbits which are homeomorphic to $T^{n}$ and the orbit space $M^{*}=M^{n+2} / T^{n}$ is a simply connected compact 2-manifold.

We first prove the following theorem as an application of a result of Pak [4]. It will say that $T^{n}$ cannot act freely on simply connected closed $(n+2)$-manifold $M^{n+2}$ unless $n=1$.

Theorem 1.1. If $T^{n}$ acts on a closed orientable $(n+2)$-manifold $M^{n+2}$ with one or two types of orbit structure, and the orbit space $M^{*}$ is simply connected, then $M^{n+2}=L(p, q) \times T^{n-1}$ for $n \geqq 2$, where $L(p, q)$ is a lens space which includes the case of $S^{3}$ and $S^{2} \times S^{1}$.

Proof. Suppose the action is free (only one orbit type). Since $M^{*}$ is simply connected it must be $S^{2}$. Let $T^{n-1} \subset T^{n}$ and $T^{1}$ be a complementary circle subgroup to $T^{n-1}$ in $T^{n}$. Let $N$ denote the orbit space $M^{n+2} / T^{n-1}$. Then $T^{1}$ acts freely on $N$ such that $N / T^{1}=S^{2}$. Then $N$ is either $S^{3}, S^{2} \times S^{1}$ or $L(|k|, 1)$ for some integer $k \in Z$. Therefore, $M^{n+2}$ is a principal $T^{n-1}$-bundle over these spaces. Then it follows by [4] that $M^{n+2}=L(p, q) \times T^{n-1}$ for $n \geqq 2$.

Now suppose that there are two types of orbit. Then $T^{i}$ alone cannot be isotropy subgroup of $T^{n}$ for a fixed $i \geqq 2$. Therefore, $T^{1}$ is the only nonempty isotropy subgroup of $T^{n}$. Then $T^{n-1}=T^{n} / T^{1}$ acts freely on $M^{n+2}$ and the orbit space $N=M^{n+2} / T^{n-1}$ is a 3-manifold on which $T^{1}$ acts semi-freely and $N / T^{1}=D^{2}$ and $\partial D^{2}=F\left(T^{1}, N\right)$. Then $N$ is the 3-sphere $S^{3}$, which is gotten from $D^{2} \times T^{1}$ by identifying each $t \times S^{1}$ to a single point for all $t \in \partial D^{2}$. Then $M^{n+2}$ is the trivial $T^{n-1}$-bundle over $S^{3}$. Thus $M^{n+2}=S^{3} \times T^{n-1}$.

COROLLARY 1.2. $T^{n}$ cannot act freely on simply connected closed $(n+2)$-manifold $M^{n+2}$ unless $n=1$.

Proof. If $T^{n}$ acts freely on $M^{n+2}$, then $M^{n+2}=L(p, q) \times T^{n-1}$ for $n \geqq 2$ so that $M^{n+2}$ is not simply connected. For $n=1$, the Hopf fibering $\left(T^{1}, S^{3}\right)$ is a free action.

We note that Corollary 1.2 can also be seen by looking at the homotopy exact sequence of the fibering $T^{n} \rightarrow M \rightarrow S^{2}$. We also note 
that $T^{n}$, for $n \geqq 2$, cannot act freely on any sphere $S^{m}$ since it contains a subgroup $Z_{p} \oplus Z_{p}$.

If $T^{n}$ acts effectively on a simply connected closed $(n+2)$-manifold $M^{n+2}$, then there must exist a nontrivial isotropy subgroup since the action cannot be free for $n \geqq 2$. It is not too hard to see that for $i \geqq 3$, any $T^{i}$ subgroup of $T^{n}$ cannot be an isotropy subgroup, and $T^{1}$ or $T^{2}$ subgroups cannot be the only isotropy subgroup of $T^{n}$. Furthermore, the product of $T^{1}$ or $T^{2}$ with a finite subgroup of the corresponding complementary subgroups cannot be an isotropy subgroup of $T^{n}$. In summary we have:

THEOREM 1.3. Let $\left(T^{n}, M^{n+2}\right)$ be an effective $T^{n}$-action on a simply connected closed $(n+2)$-manifold $M^{n+2}, n \geqq 2$. Then both $T^{1}$ and $T^{2}$ subgroups of $T^{n}$ must appear as isotropy subgroups of $T^{n}$ and these are the only possible nontrivial isotropy subgroups of $T^{n}$, and the $T^{1}$ 's are subgroups of the $T^{2}$ 's, and at least two $T^{2}$ subgroups always appear. If $n \geqq 3$, then three or more $T^{2}$ subgroups must appear as isotropy subgroups of $T^{n}$ (see the next example). Hence the orbit space $M^{*}$ is a disk $D^{2}$ with the boundary points, $\partial D^{2}$, corresponding to only $T^{1}$ 's and $T^{2}$ 's, and the interior points corresponding to only principal orbits.

For $n=2$, the theorem says that $F\left(T^{2}, M^{4}\right) \neq \varnothing$ for simply connected 4-dimensional manifolds, thus generalizing the result [3, I, Lemma 5.1].

Proof of this theorem uses the parity of the dimensions of the slice and that of the orbit. We leave the proof to the reader.

EXAMPLE 1.4. Let the orbit space $M^{*}$ of an effective action $\left(T^{n}\right.$, $M^{n+2}$ ) be given by $D^{2}$ and isotropy subgroups on $\partial D^{2}=S^{1}$ are given as on the figure:

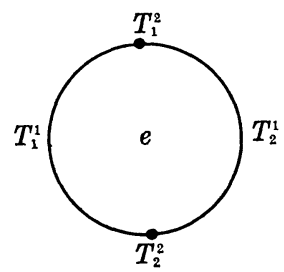

In this case $T_{1}^{1} \cup T_{2}^{1}$ generates $T_{1}^{2}$ and $T_{2}^{2}$ so that $T_{1}^{2}=T_{2}^{2}$. Let $T^{n-2}$ be the complementary subgroup of $T_{1}^{2}$ in $T^{n}$. Then $M^{n+2}$ is a principal $T^{n-2}$-bundle over $M^{n+2} / T^{n-2}$ which is a 4 -manifold. Then by [3, (5.4)] $M^{n+2} / T^{n-2}=S^{4}$. Since these bundles are classified by $H^{2}\left(S^{4} ; \bigoplus_{1}^{n-1} Z\right)=$ 0 , it is a trivial bundle. Then $M^{n+2}=S^{4} \times T^{n-2}$, which is not simply 
connected for $n \geqq 3$.

We also prove the existence of a particular isotropy subgroup $T^{1}$ in $T^{n}$, which fixes a nonsimply connected subspace of $M^{n+2}$.

THEOREM 1.5. If $T^{n}$ acts effectively on a simply connected closed $(n+2)$-manifold $M^{n+2}$, then there exists a circle subgroup $T^{1}$ in $T^{n}$ such that every component of $F\left(T^{1}, M^{n+2}\right)$ is homeomorphic to $L_{3}(p, q) \times T^{n-3}$ for $n \geqq 4$.

Proof. We know that $M^{*}=M^{n+2} / T^{n}$ is a disk $D^{2}$ and each point of $\partial D^{2}$ corresponds to either an orbit of an isotropy subgroup $T^{1} \subset T^{n}$ or $T^{2} \subset T^{n}$ and $T^{1} \subset T^{2}$ for some $T^{2} \subset T^{n}$. Then $T^{1}$ acts on $M^{n+2}$ semi-freely and $M^{n+2} / T^{1}$ is a simply connected $(n+1)$-manifold $M^{n+1}$ with boundary $\partial\left(M^{n+1}\right)=F\left(T^{1}, M^{n+2}\right)$. Let $M^{n}$ be a component of $\partial\left(M^{n+1}\right)$. Now $T^{n-1}$, the complementary subgroup of $T^{1}$ in $T^{n}$, acts on $M^{n}$ and the orbit space $M^{n} / T^{n-1}$ is homeomorphic to the unit interval $[0,1]$ since $T^{1} \subset T^{2}$ and the complementary subgroup of $T^{1}$ in $T^{2}$ is a subgroup of $T^{n-1}$. From the results of [4], we conclude that $M^{n}=L_{3}(p, q) \times T^{n-3}$ for $n \geqq 4$. This completes the proof.

It is easy to see, by using the result of [3], that if the number of $T^{2}$ stability subgroups is small and there exists a $T^{n-2}$ subgroup which is disjoint from all stability subgroups then the action ( $T^{n}$, $M^{n+2}$ ) is not effective. Furthermore, we actually prove that all $T^{2}$ stability subgroups must generate the whole group $T^{n}$.

THEOREM 1.6. If $\left(T^{n}, M^{n+2}\right)$ is an effective action of a torus group $T^{n}$ on a simply connected closed $(n+2)$-manifold $M^{n+2}$, then no subgroup $T^{k}(n>k \geqq 1)$ can contain all nonfree elements of $T^{n}$.

Proof. Suppose a subgroup $T^{k}$ contains all nonfree elements of $T^{n}$. If $T^{n-k}$ is the complementary subgroup of $T^{k}$ in $T^{n}$ then $T^{n-k}$ acts freely on $M^{n+2}$. Thus we have the commutative triangle:

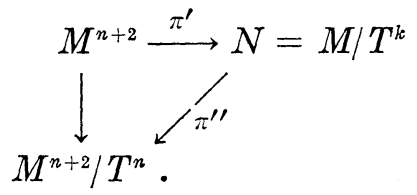

The orbit space $N$ relative to a $T^{k}$-action on $M^{n+2}$ is simply connected and $T^{n-k}$ acts freely on the space $N$. For if there is an element $g \in T^{n-k}$ which fixes an element $\bar{x} \in N$, i.e., $g \bar{x}=\bar{x}$, then $\overline{g x}=\bar{x}$. Therefore, there exists an element $h_{x} \in T^{k}$ such that $g x=h_{x} x$. Then $h_{x}^{-1} g$ fixes the element $x$, and hence $h_{x}^{-1} g \in T^{k}$. This implies that $g \in T^{k} \cap$ $T^{n-k}$. Thus $g=e$. Therefore, $\pi^{\prime \prime}: N \rightarrow M^{n+2} / T^{n}$ is a principal $T^{n-k}$ - 
bundle. Since the space $M^{n+2} / T^{n}$ is a disk, $N$ is homeomorphic to $D^{2} \times T^{n-k}$, contradicting the fact that $N$ is simply connected.

COROLlaRY 1.7. If $\left(T^{n}, M^{n+2}\right)$ is an effective action of a torus group $T^{n}$ on a simply connected closed $(n+2)$-manifold $M^{n+2}$, then all stability subgroups must generate the whole group $T^{n}$, and there are at least $n$ different circle stability subgroups of $T^{n}$.

As illustrations of the above fact there are $T^{2}$-actions on simply connected 4-manifolds (see [3]). Also we have the action $\left(T^{3}, S^{5}\right)$ given by $\left(t_{1}, t_{2}, t_{3}\right)\left(z_{1}, z_{2}, z_{3}\right)=\left(t_{1} z_{1}, t_{2} z_{2}, t_{3} z_{3}\right),\left(t_{1}, t_{2}, t_{3}\right) \in T^{1} \times T^{1} \times T^{1}=$ $T^{3},\left(z_{1}, z_{2}, z_{3}\right) \in S^{5} \subset C^{3}$, and the actions $\left(T^{2} \times T^{2}, S^{2} \times S^{3}\right)$ and $\left(T^{2} \times T^{2}\right.$, $\left.S^{3} \times S^{3}\right)$.

2. Cross-sectioning theorem and equivariant classification. In [3], Orlik and Raymond proved a cross-sectioning theorem for the orbit map $\pi: M^{n+2} \rightarrow M^{*}=M^{n+2} / T^{n}$ for $n=2$. By the similar technique we can prove a cross-sectioning theorem for all $n \geqq 2$. To see this, we use the following two lemmas. The proofs are slight generalizations of those in [3].

Lemma 2.1. Let $\left(T^{n}, M^{n+2}\right)$ be an effective $T^{n}$-action on an $(n+$ 2)-manifold such that the orbit space $M^{*}$ is $[0,1] \times[0,1]$ and such that there are only two types of orbits. Suppose that the points on the arc $[0,1] \times 0$ have stability subgroup $T^{1} \subset T^{n}$ and all other points correspond to principal orbits $T^{n}$. Then the orbit map $\pi: M^{n+2} \rightarrow$ $M^{*}$ has a cross-section. Moreover, every cross-section given on the arc $A=(0 \times[0,1]) \cup([0,1] \times 1) \cup(1 \times[0,1])$ may be extended to $a$ cross-section over all of $M^{*}$.

LEMma 2.2. Let $\left(T^{n}, M^{n+2}\right)$ be an effective $T^{n}$-action on an $(n+2)$ manifold $M^{n+2}$. Assume that the orbit space $M^{*}$ is $[-1,1] \times[0,1]$ and that the points on $[-1,0) \times 0$ have stability subgroup $T_{1}^{1} \subset T^{n}$, the points on $(0,1] \times 0$ have stability subgroup $T_{2}^{1} \subset T^{n}$, the point $0 \times 0$ has stability subgroup $T^{2}$ which is generated by $T_{1}^{1}$ and $T_{2}^{1}$, and all other points correspond to principal orbits $T^{n}$. Then there is a cross-section for the orbit map $\pi: M^{n+2} \rightarrow M^{*}$. Moreover, any cross-section on the arc $A=((-1) \times[0,1]) \cup([-1,1] \times 1) \cup(1 \times[0,1])$ may be extended to a cross-section over all of $M^{*}$.

Now we state a cross-sectioning theorem for an effective $T^{n}$-action on a simply connected closed $(n+2)$-manifold $M^{n+2}$.

TheOREM 2.3. Let $\left(T^{n}, M^{n+2}\right)$ be an effective $T^{n}$-action on a simply 
connected closed $(n+2)$-manifold $M^{n+2}$. Then the orbit map $\pi: M^{n+2} \rightarrow$ $M^{*}$ has a cross-section.

Now we know the complete orbit structures and the existence of cross-sections for the orbit maps $\pi: M^{n+2} \rightarrow M^{*}$ for effective $T^{n}$ actions on simply connected closed $(n+2)$-manifolds. Therefore, we can classify equivariantly these simply connected closed $(n+2)$-manifolds which admit effective $T^{n}$-actions.

Let $M^{*}$ be the orbit space of an action $\left(T^{n}, M^{n+2}\right)$. We associate the stability subgroup of the points in the orbit to each orbit $x^{*} \in M^{*}$. The orbit space $M^{*}$ with associated orbit types is called a weighted orbit space.

Let $M^{*}$ and $N^{*}$ denote the weighted orbit spaces of a $T^{n}$-action on $M^{n+2}$ and $N^{n+2}$, respectively. A weight preserving homeomorphism of $M^{*}$ onto $N^{*}$ is a homeomorphism of $M^{*}$ onto $N^{*}$ which carries the weights of $M^{*}$ isomorphically onto the weights of $N^{*}$.

If manifolds $M^{n+2}$ and $N^{n+2}$ are simply connected and closed then the orbit spaces $M^{*}$ and $N^{*}$ are disks with nontrivial stability subgroups $T^{1}$ 's and $T^{2}$ 's on the boundaries and the orbit map $\pi: N \rightarrow$ $N^{*}$ have cross-sections by the above Theorem 2.3. By the same argument that is used in [3] and [5], we have an equivariant classification theorem.

THEOREM 2.4. Let $T^{n}$ act effectively on the simply connected closed $(n+2)$-manifolds $M^{n+2}$ and $N^{n+2}$. Then there is an equivariant homeomorphism $f$ of $M$ onto $N$ if and only if there is a weight preserving homeomorphism $f^{*}$ of $M^{*}$ onto $N^{*}$.

3. Free actions. We know by [4] and from the previous section that a torus group $T^{n}$ cannot act freely on a simply connected $(n+1)$ or $(n+2)$-manifold for $n \geqq 3$ or $n \geqq 2$, respectively. This is of course not true the higher codimension. For example for the codimension 4, the action $\left(T^{2}, S^{3} \times S^{3}\right)$ induced by the Hopf fibering $\left(T^{1}, S^{3}\right)$ is free. We note that this action $\left(T^{2}, S^{3} \times S^{3}\right)$ has the orbit space $S^{2} \times S^{2}$, and by taking equivariant connected sums of $S^{3} \times S^{3}$ 's we get more free $T^{2}$-actions on simply connected closed 6-manifolds. For all $n$, we demonstrate the existence of a principal $T^{n-2}$-bundle over a connected sum of several copies of $S^{2} \times S^{2}$ with a simply connected total space.

Let $X$ be the connected sum of $k$ copies of $S^{2} \times S^{2}$, i.e., $X=$ $\#_{i=1}^{k}\left(S^{2} \times S^{2}\right)_{i}$. Then we know $H_{1}(X, Z)=0$ and $H_{2}(X, Z)=\bigoplus_{1}^{2 k} Z=Z^{2 k}$. Hence $\pi_{2}(X) \cong Z^{2 k}$. Moreover, the universal-coefficient theorem [7] shows that we have an isomorphism $h: H^{2}\left(X, Z^{n-2}\right) \rightarrow \operatorname{Hom}\left(H_{2}(X, Z), Z^{n-2}\right)$ defined by $h(f)\left(\Sigma c_{i}\right)=\Sigma f\left(c_{i}\right)$ where $f$ and $\Sigma c_{i}$ represent cohomology 
and homology classes, respectively.

Let $P^{\prime}: E^{\prime} \rightarrow B_{T^{n-2}}$ be a universal $T^{n-2}$-bundle where the classifying space $B_{T^{n-2}}$ is the product of $n-2$ copies of the Eilenberg-MacLane space $K(Z, 2)$. Then principal $T^{n-2}$-bundles over $X$ are in a one-toone correspondence with the set of homotopy classes of maps from $X$ to $B,[X, B]$.

We also have a bijection $\psi:[X, B] \rightarrow H^{2}\left(X, Z^{n-2}\right)$ defined as follows. Let $\alpha \in H^{2}\left(B, Z^{n-2}\right)$ be 2-characteristic, i.e., $h(\alpha): H_{2}(B, Z) \rightarrow Z^{n-2}$ is an isomorphism, where $h$ again comes from the universal-coefficient theorem. Then $\psi[f]=f^{*}(\alpha) \in H^{2}\left(X, Z^{n-2}\right)$ where $[f]$ is a class in $[X$, $B]$ with a representative $f$.

Let $\varphi_{X}: \pi_{2}(X) \rightarrow H_{2}(X, Z)$ and $\varphi_{B}: \pi_{2}(B) \rightarrow H_{2}(B, Z)$ be the Hurewicz isomorphisms. Then we have an obvious isomorphism $\gamma: \operatorname{Hom}\left(H_{2}(X\right.$, $\left.Z), Z^{n-2}\right) \rightarrow \operatorname{Hom}\left(\pi_{2}(X), \pi_{2}(B)\right)$ defined by: $\gamma(g)=\varphi_{B}^{-1} \circ(h(\alpha))^{-1} \circ g \circ \varphi_{X}$.

LEMmA 3.1. $[X, B] \cong \operatorname{Hom}\left(\pi_{2}(X), \pi_{2}(B)\right)$ where the image of $a$ class $[f] \in[X, B]$ is $f_{\sharp}: \pi_{2}(X) \rightarrow \pi_{2}(B)$.

Proof. From the above comments we have $[X, B] \cong \operatorname{Hom}\left(\pi_{2}(X)\right.$, $\left.\pi_{2}(B)\right)$ by the map $\gamma \circ h \circ \psi$. We must show that $\gamma \circ h \circ \psi[f]=f_{\sharp}$.

Let $\sigma: S^{2} \rightarrow X$ be a representative map of an element $[\sigma] \in \pi_{2}(X)$. We have $\gamma[h \circ \psi([f])]([\sigma])=\varphi_{B}^{-1} \circ(h(\alpha))^{-1} \circ(h \circ \psi([f])) \varphi_{X}([\sigma])$. And we have $\varphi_{B}^{-1} \circ(h(\alpha))^{-1} h\left(f^{*}(\alpha)\right) \varphi_{X}([\sigma])=\varphi_{B}^{-1}(h(\alpha))^{-1} f^{*}(\alpha) \varphi_{X}([\sigma])$

$$
\begin{aligned}
& =\varphi_{B}^{-1}(h(\alpha))^{-1} \alpha f_{*} \varphi_{X}([\sigma]) \\
& =\varphi_{B}^{-1}(h(\alpha))^{-1} \alpha\left(\varphi_{B} f_{\#}([\sigma])\right)
\end{aligned}
$$

by the commutative diagram

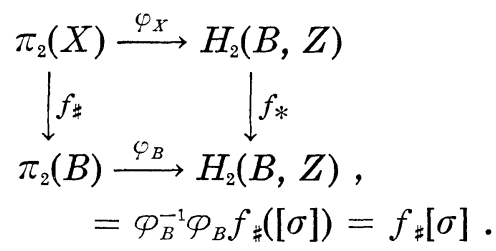

This completes the proof.

THEOREM 3.2. Let $X$ be the connected sum of $k$ copies of $S^{2} \times$ $S^{2}$, where $k \geqq n / 2-1$. Then there is a principal $T^{n-2}$-bundle over $X$ with a simply connected total space.

Proof. Choose an element $f_{\#}$ in $\operatorname{Hom}\left(\pi_{2}(X), \pi_{2}(B)\right) \cong \operatorname{Hom}\left(Z^{2 k}\right.$, $Z^{n-2}$ ) which is onto. The existence of an onto map is guaranteed by the choice of $k$. Let $p: E \rightarrow X$ be the $T^{n-2}$-bundle induced by $f: X \rightarrow$ $B$. Then $f$ induces a map between the homotopy sequences for 
bundles $p^{\prime}: E^{\prime} \rightarrow B$ and $p: E \rightarrow X$. Thus we have the following commutative diagram

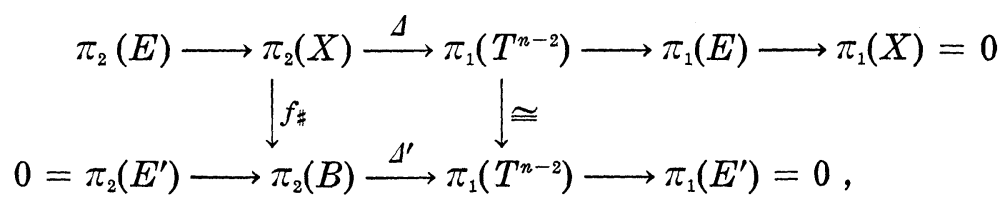

where the rows are exact and $\pi_{2}\left(E^{\prime}\right)=0=\pi_{1}\left(E^{\prime}\right)$ since $E^{\prime}$ is contractible. Therefore $A^{\prime}$ is an isomorphism. Since $f_{\#}$ is chosen to be onto, $\Delta: \pi_{2}(X) \rightarrow \pi_{1}\left(T^{n-2}\right)$ must be onto. Hence $\pi_{1}(E)=0$. This completes the proof.

4. Effective actions. Now we study effective actions of the codimension 2. There are many $(n+2)$-manifolds which do not allow any $T^{n}$-action:

4.1. [D. Montgomery]. $T^{n}$ cannot act effectively on the $(n+2)-$ sphere $S^{n+2}$ for $n>3$, or on $R^{n+2}$ for $n>2$.

4.2. $T^{2 n}$ cannot act effectively on $S^{p} \times S^{q}$ with $p, q$ odd and $p+q=2 n+2$ for $n>2$.

4.1. follows from Borel's formula, and 4.2 can be deduced from Golber's formula.

We know that $T^{n}$ cannot act effectively on a simply connected $(n+1)$-manifold for $n \geqq 3$ [4], and we suspected that $T^{n}$ cannot act effectively on a simply connected $(n+2)$-manifold for $n \geqq 5$. However, contrary to our suspicion, we construct an effective $T^{n}$-action on a simply connected $(n+2)$-manifold for any $n$.

We first define certain permutations. Let $\sigma_{k}=(12 k+1)$ for $2 \leqq$ $k<n$ and $\sigma_{n}=(123)$. Let $\mu_{1}=(1)$ and for $1<k \leqq n$ define $\mu_{k}$ by $\mu_{k} \circ \sigma_{k}=\mu_{k-1}$ (where $\mu_{k} \circ \sigma_{k}$ means " $\sigma_{k}$ followed by $\mu_{k}^{\prime \prime}$ ). Finally define $\sigma_{n+1}$ by $\mu_{n} \circ \sigma_{n+1}=(1)$. Let $D^{4} \times T^{n-2}=\left\{\left(t_{1}, t_{2}, \cdots, t_{n}\right) \mid\left(t_{1}, t_{2}\right) \in\right.$ $\left.D^{2} \times D^{2}=D^{4}\right\}$. We shall consider various copies of $D^{4} \times T^{n-2}$ and we will denote the $k$ th copy by $\left(D^{4} \times T^{n-2}\right)_{k}$. Note that $\partial\left(D^{4} \times T^{n-2}\right)_{k}=$ $\left(S^{1} \times D^{2} \times T^{n-2}\right)_{k} \cup\left(D^{2} \times S^{1} \times T^{n-2}\right)_{k}$.

For each $2 \leqq k \leqq n$ define $f_{k}:\left(S^{1} \times D^{2} \times T^{n-2}\right)_{k} \rightarrow\left(D^{2} \times S^{1} \times\right.$ $\left.T^{n-2}\right)_{k-1}$ by $f_{k}\left(t_{1}, \cdots, t_{n}\right)=\left(t_{\sigma_{k}(1)}, \cdots, t_{\sigma_{k}(n)}\right)$. Note that for $2 \leqq k<n$, $f_{k}\left(t_{1}, \cdots, t_{n}\right)=\left(t_{2}, t_{k+1}, t_{3}, \cdots, t_{1}, \cdots, t_{n}\right)$ with $t_{1}$ appearing in the $(k+$ 1)st position and for $k=n f_{n}\left(t_{1}, \cdots, t_{n}\right)=\left(t_{2}, t_{3}, t_{1}, \cdots, t_{n}\right)$. Hence each $f_{k}$ is a homeomorphism.

For $k=n+1$ define $f_{n+1}:\left(D^{2} \times S^{1} \times T^{n-2}\right)_{n} \rightarrow\left(S^{1} \times D^{2} \times T^{n-2}\right)_{1}$ by $f_{n+1}\left(t_{1}, \cdots, t_{n}\right)=\left(t_{\sigma_{n+1}(1)}, \cdots, t_{\sigma_{n+1}(n)}\right)$. We must show that $f_{n+1}$ is a homeomorphism, i.e., we must show that $\sigma_{n+1}(2)=1$.

LEMMA 4.3. $\sigma_{n} \circ \sigma_{n-1} \circ \cdots \circ \sigma_{3} \circ \sigma_{2}=\sigma_{n+1}$. 
Proof. $\sigma_{i}=\mu_{i}^{-1} \circ \mu_{i-1}$ for $2 \leqq i \leqq n$ and $\sigma_{n+1}=\mu_{n}^{-1}$. Hence

$$
\begin{aligned}
\sigma_{n} \circ \cdots \circ \sigma_{2} & =\mu_{n}^{-1} \circ \mu_{n-1} \circ \mu_{n-1}^{-1} \circ \mu_{n-2} \circ \cdots \circ \mu_{3}^{-1} \circ \mu_{2} \circ \mu_{2}^{-1} \circ \mu_{n} \\
& =\mu_{n}^{-1} \circ \mu_{1}=\sigma_{n+1} .
\end{aligned}
$$

Now computations show that $\sigma_{n+1}(2)=1$ and hence $f_{n+1}$ is a homeomorphism.

For each $1 \leqq k \leqq n$ define a $T^{n}$-action on $\left(D^{4} \times T^{n-2}\right)_{k}$ by $\left(\tau_{1}\right.$, $\left.\cdots, \tau_{n}\right) \times\left(t_{1}, \cdots, t_{n}\right)=\left(\tau_{\mu_{k}(1)} t_{1}, \cdots, \tau_{\mu_{k}(n)} t_{n}\right)$.

LEMMA 4.4. The homeomorphisms $f_{k}(2 \leqq k \leqq n)$ and $f_{n+1}$ are equivariant with respect to this action.

Proof. For $2 \leqq k \leqq n, \tau \in T^{n}$ and $t \in\left(D^{4} \times T^{n-2}\right)_{k}$ we have

$$
\begin{aligned}
f_{k}(\tau \times t) & =f_{k}\left(\tau_{\mu_{k}(1)} t_{1}, \tau_{\mu_{k}(2)} t_{2}, \cdots, \tau_{\mu_{k}(n)} t_{n}\right) \\
& =\left(\tau_{\left(\mu_{\left.k^{\circ} \sigma_{k}\right)(1)}\right)} t_{\sigma_{k}(1)}, \cdots, \tau_{\left(\mu_{\left.k^{\circ} \sigma_{k}\right)(n)}\right.} t_{\sigma_{k}(n)}\right) \\
& \left.=\tau_{\mu_{k-1}(1)} t_{\sigma_{k}(1)}, \cdots, \tau_{\mu_{k-1}(n)} t_{\sigma_{k}(n)}\right) \\
& =\tau \times\left(t_{\sigma_{k}(1)}, \cdots, t_{\sigma_{k}(n)}\right) \\
& =\tau f_{k}\left(t_{1}, \cdots, t_{n}\right) .
\end{aligned}
$$

Hence $f_{k}$ is equivariant. Similar calculations show that $f_{n+1}$ is equivariant.

Now let $M_{1}=\left(D^{4} \times T^{n-2}\right)_{1}$ and $M_{2}=M_{1} \bigcup_{f_{2}}\left(D^{4} \times T^{n-2}\right)_{2}$. We know that $\pi_{1}\left(D^{4} \times T^{n-2}\right)_{j}=Z^{n-2}=\left\langle t_{3}\right\rangle \times \cdots \times\left\langle t_{n}\right\rangle$ where $T^{n-2}=T_{3} \times T_{4} \times$ $\cdots \times T_{n}$ and $\left\langle t_{k}\right\rangle$ corresponds to $\pi_{1}\left(T_{k}\right)$. Now we may assume that $M_{2}$ was obtained by attaching $S^{1} \times D^{2} \times T^{n-2} \times I$ to $\left(D^{2} \times S^{1} \times T^{n-2}\right)_{1} \subseteq$ $M_{1}$ via $f_{2}$ defined on $S^{1} \times D^{2} \times T^{n-2} \times 0$ and attaching $\left(D^{4} \times T^{n-2}\right)_{2}$ to $S^{1} \times D^{2} \times T^{n-2} \times 1 \leqq S^{1} \times D^{2} \times T^{n-2} \times I$ via the identity on $\left(S^{1} \times D^{2} \times T^{n-2}\right)_{2}$. For convenience we may assume $M_{1}$ and $\left(D^{4} \times\right.$ $\left.T^{n-2}\right)_{2}$ are open subsets of $M_{2}$ with $M_{1} \cap\left(D^{4} \times T^{n-2}\right)_{2}=S^{1} \times D^{2} \times$ $T^{n-2} \times I^{-}\left(I^{-}\right.$an open interval in $\left.I\right)$. Now $\pi_{1}\left(S^{1} \times D^{2} \times T^{n-2}\right) \cong$ $Z^{n-1}=\left\langle t_{1}\right\rangle \times\left\langle t_{3}\right\rangle \times \cdots \times\left\langle t_{n}\right\rangle$. If

$$
\begin{aligned}
& \varphi_{1}: \pi_{1}\left(M_{1} \cap\left(D^{4} \times T^{n-2}\right)_{2}\right) \longrightarrow \pi_{1}\left(M_{1}\right) \text { and } \\
& \varphi_{2}: \pi_{1}\left(M_{1} \cap\left(D^{4} \times T^{n-2}\right)_{2}\right) \longrightarrow \pi_{1}\left(D^{4} \times T^{n-2}\right)_{2}
\end{aligned}
$$

are the homomorphisms induced by inclusions, it is easy to see that $\operatorname{ker} \varphi_{1}=\left\langle t_{3}\right\rangle$ and $\operatorname{ker} \varphi_{2}=\left\langle t_{1}\right\rangle$ and that each $\varphi_{i}$ is onto. Then by applying Van Kampen's theorem it is also easy to see that $\pi_{1}\left(M_{2}\right)$ is isomorphic to $\pi_{1}\left(M_{1} \cap\left(D^{4} \times T^{n-2}\right)_{2}\right)$ modulo the smallest normal subgroup containing $\left(\operatorname{ker} \varphi_{1}\right) \cup\left(\operatorname{ker} \varphi_{2}\right)$, i.e., $\pi_{1}\left(M_{2}\right) \cong Z^{n-3}=\left\langle t_{4}\right\rangle \times \cdots \times\left\langle t_{n}\right\rangle$.

Now let $M_{3}=M_{2} \bigcup_{f_{3}}\left(D^{4} \times T^{n-3}\right)_{3}$ where we consider $f_{3}$ as a mapping onto $\left(D^{2} \times S^{1} \times T^{n-2}\right)_{2} \subseteq\left(D^{4} \times T^{n-2}\right)_{2} \subseteq M_{2}$. The same argument shows that $\pi_{1}\left(M_{3}\right) \cong Z^{n-4}$. Continuing this process we obtain 
LEMMA 4.5. $\quad M_{n-1}=M_{n-2} \bigcup_{f_{n-1}}\left(D^{4} \times T^{n-2}\right)_{n-1}$ is a simply connected manifold with the boundary $\partial M_{n-1}=\left(D^{2} \times S^{1} \times T^{n-2}\right)_{n-1} \cup\left(S^{1} \times D^{2} \times\right.$ $\left.T^{n-2}\right)_{1}$.

$$
\begin{aligned}
& \text { Define } f: \partial\left(D^{4} \times T^{n-2}\right)_{n} \rightarrow \partial M_{n-1} \text { by } \\
& \qquad f\left(t_{1}, \cdots, t_{n}\right)=\left\{\begin{array}{l}
f_{n}\left(t_{1}, \cdots, t_{n}\right) \text { for } t \in\left(S^{1} \times D^{2} \times T^{n-2}\right)_{n} \\
f_{n+1}\left(t_{1}, \cdots, t_{n}\right) \text { for } t \in\left(D^{2} \times S^{1} \times T^{n-2}\right)_{n} .
\end{array}\right.
\end{aligned}
$$

Then these definitions agree on $\left(S^{1} \times D^{2} \times T^{n-2}\right)_{n} \cap\left(D^{2} \times S^{1} \times T^{n-2}\right)_{n}=$ $\left(S^{1} \times S^{1} \times T^{n-2}\right)_{n}$. For if $t \in\left(S^{1} \times S^{1} \times T^{n-2}\right)_{n}$, on the one hand, we have $t$ identified with $\left(f_{2} \circ \cdots \circ f_{n}\right)(t)=\left(t_{\sigma_{n^{0} \cdots \sigma_{2}(1)}}, \cdots, t_{\left(\sigma_{n} \cdots \sigma_{2}\right)(n)}\right) \in\left(S^{1} \times\right.$ $\left.S^{1} \times T^{n-2}\right)_{1} \leqq M_{n-1}$. On the other hand, $t$ is identified with $f_{n+1}(t)=$ $\left(t_{\sigma_{n+1}(1)}, \cdots, t_{\sigma_{n+1}(n)}\right) \in\left(S^{1} \times S^{1} \times T^{n-2}\right)_{1} \subseteq M_{n-1}$. Then Lemma 4.1 shows these two are equal.

LEMмA 4.6. The space $M_{n}=M_{n-1} \bigcup_{f}\left(D^{4} \times T^{n-2}\right)_{n}$ is a simply connected closed $(n+2)$-manifold.

Proof. Since $f$ is a homeomorphism from $\partial\left(D^{4} \times T^{n-2}\right) n$ onto $\partial M_{n-1}, M_{n}$ is a compact manifold without boundary. It is easy to see that the homomorphism $\varphi: \pi_{1}\left(\partial\left(D^{4} \times T^{n-2}\right)_{n}\right) \rightarrow \pi_{1}\left(D^{4} \times T^{n-2}\right)_{n}$ induced by the inclusion is onto. A further application of Van Kampen's theorem shows that $M_{n}$ is simply connected.

Therefore we have proved the following:

THEOREM 4.7. There exists an effective $T^{n}$-action on a simply connected closed $(n+2)$-manifold $M^{n+2}$.

\section{REFERENCES}

1. G. Bredon, Introduction to Compact Transformation Groups, Academic Press, N. Y., 1972.

2. D. Montgomery and C. T. Yang, The existence of a slice, Ann. of Math., 65 (1957), 108-116.

3 P. Orlik and F. Raymond, Actions of the torus on 4-manifolds, I and II, I-Trans. Amer. Math. Soc., 152 (1970), 531-559; II-to appear.

4. J. Pak, Actions of torus $T^{n}$ on $(n+1)$-manifolds $M^{n+1}$, Pacific J. Math, 44 (1973), 671-674.

5. R. Richardson, Jr., Groups acting on the 4-sphere, Illinois J. Math., 5 (1961), 474485.

6. E. H. Spanier, Algebraic Topology, McGraw-Hill, N. Y., 1966.

7. N. Steenrod, The Topology of Fiber Bundles, Princeton Univ. Press, N. J., 1951.

Received April 5, 1973 and in revised form February 15, 1974. Soon-Kyu Kim was partially supported by Grant NSF GP-29117. Dennis McGavran was partially supported by a NSF Traineeship.

The University of Connecticut

AND

Wayne State University 


\section{PACIFIC JOURNAL OF MATHEMATICS}

\section{EDITORS}

RICHARD ARENS (Managing Editor)

University of California

Los Angeles, California 90024

\section{J. DUGUNDJI}

Department of Mathematics University of Southern California Los Angeles, California 90007

D. Gilbarg and J. Milgram

Stanford University

Stanford, California 94305

University of Washington
Seattle, Washington 98105

ASSOCIATE EDITORS
E. F, BECKENBACH
B. H. NEUMANN
F. WOLF
K. Yoshida

\section{SUPPORTING INSTITUTIONS}

\author{
UNIVERSITY OF BRITISH COLUMBIA \\ CALIFORNIA INSTITUTE OF TECHNOLOGY \\ UNIVERSITY OF CALIFORNIA \\ MONTANA STATE UNIVERSITY \\ UNIVERSITY OF NEVADA \\ NEW MEXICO STATE UNIVERSITY \\ OREGON STATE UNIVERSITY \\ UNIVERSITY OF OREGON \\ OSAKA UNIVERSITY
}

\author{
UNIVERSITY OF SOUTHERN CALIFORNIA \\ STANFORD UNIVERSITY \\ UNIVERSITY OF TOKYO \\ UNIVERSITY OF UTAH \\ WASHINGTON STATE UNIVERSITY \\ UNIVERSITY OF WASHINGTON \\ * * * * \\ AMERICAN MATHEMATICAL SOCIETY \\ NAVAL WEAPONS CENTER
}

The Supporting Institutions listed above contribute to the cost of publication of this Journal, but they are not owners or publishers and have no responsibility for its content or policies.

Mathematical papers intended for publication in the Pacific Journal of Mathematics should be in typed form or offset-reproduced, (not dittoed), double spaced with large margins. Underline Greek letters in red, German in green, and script in blue. The first paragraph or two must be capable of being used separately as a synopsis of the entire paper. Items of the bibliography should not be cited there unless absolutely necessary, in which case they must be identified by author and Journal, rather than by item number. Manuscripts, in duplicate if possible, may be sent to any one of the four editors. Please classify according to the scheme of Math. Rev. Index to Vol. 39. All other communications to the editors should be addressed to the managing editor, or Elaine Barth, University of California, Los Angeles, California, 90024.

100 reprints are provided free for each article, only if page charges have been substantially paid. Additional copies may be obtained at cost in multiples of 50 .

The Pacific of Journal Mathematics is issued monthly as of January 1966. Regular subscription rate: $\$ 72.00$ a year (6 Vols., 12 issues). Special rate: $\$ 36.00$ a year to individual members of supporting institutions.

Subscriptions, orders for back numbers, and changes of address should be sent to Pacific Journal of Mathematics, 103 Highland Boulevard, Berkeley, California, 94708.

PUBLISHED BY PACIFIC JOURNAL OF MATHEMATICS, A NON-PROFIT CORPORATION

Printed at Kokusai Bunken Insatsusha (International Academic Printing Co., Ltd.), 270, 3-chome Totsuka-cho, Shinjuku-ku, Tokyo 160, Japan.

Copyright (C) 1973 by Pacific Journal of Mathematics Manufactured and first issued in Japan 


\section{Pacific Journal of Mathematics}

\section{Vol. 53, No. $2 \quad$ April, 1974}

Kenneth Abernethy, On characterizing certain classses of first countable spaces by

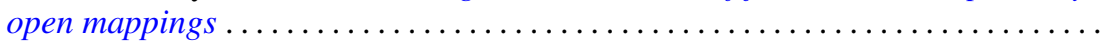

Ross A. Beaumont and Donald Lawver, Strongly semisimple abelian groups .......

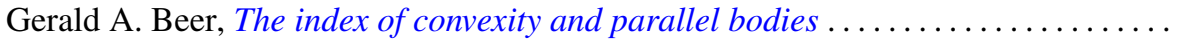

Victor P. Camillo and Kent Ralph Fuller, On Loewy length of rings ..............

Stephen LaVern Campbell, Linear operators for which $T^{*} T$ and $T T^{*}$ commute.

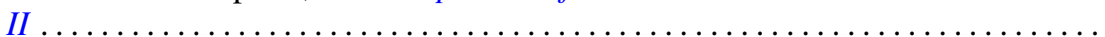

Charles Kam-Tai Chui and Philip Wesley Smith, Characterization of a function by

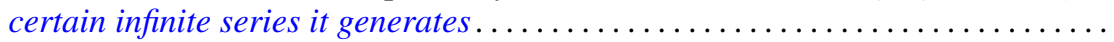

Allan L. Edelson, Conjugations on stably almost complex manifolds . ...........

Patrick John Fleury, Hollow modules and local endomorphism rings . . ..........

Jack Tilden Goodykoontz, Jr., Connectedness im kleinen and local connectedness in

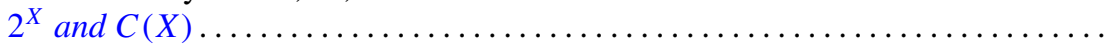

Robert Edward Jamison, II, Functional representation of algebraic intervals .......

Athanassios G. Kartsatos, Nonzero solutions to boundary value problems for

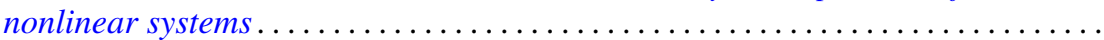

Soon-Kyu Kim, Dennis McGavran and Jingyal Pak, Torus group actions on simply

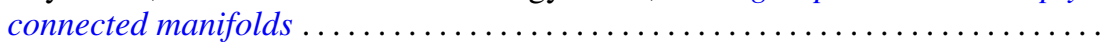

David Anthony Klarner and R. Rado, Arithmetic properties of certain recursively

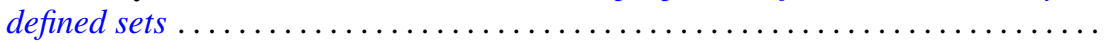

Ray Alden Kunze, On the Frobenius reciprocity theorem for square-integrable

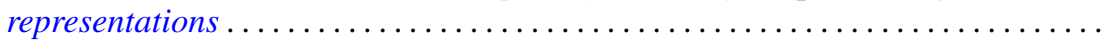

John Lagnese, Existence, uniqueness and limiting behavior of solutions of a class of differential equations in Banach space...

Teck Cheong Lim, A fixed point theorem for families on nonexpansive mappings Lewis Lum, A quasi order characterization of smooth continua

Andy R. Magid, Principal homogeneous spaces and Galois extensions . .

Charles Alan McCarthy, The norm of a certain derivation ..... . .

Louise Elizabeth Moser, On the impossibility of obtaining $S^{2} \times S^{1}$ by elementary surgery along a knot. .

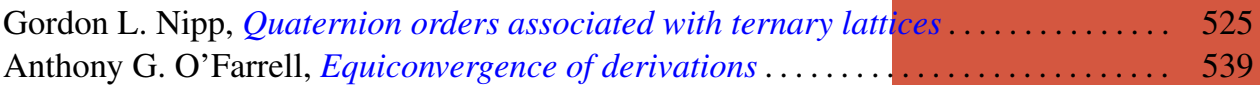

Dorte Olesen, Derivations of $A W^{*}$-algebras are inner . . . . . . . . . . . . . . . 555

Dorte Olesen and Gert Kjærgaard Pedersen, Derivations of $C^{*}$-algebras have

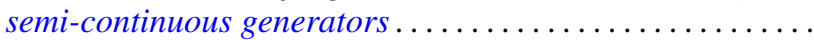

Duane O’Neill, On conjugation cobordism.

Chull Park and S. R. Paranjape, Probabilities of Wiener paths crossing differentiable

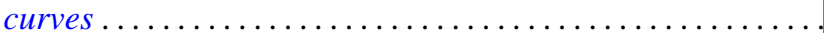

Edward Ralph Rozema, Almost Chebyshev subspaces of $L^{1}(\mu$;

Lesley Millman Sibner and Robert Jules Sibner, A note on the Atiyah-Bott fixed

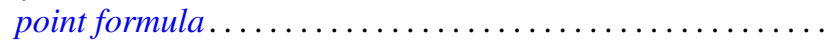

Betty Salzberg Stark, Irreducible subgroups of orthogonal groups generated by

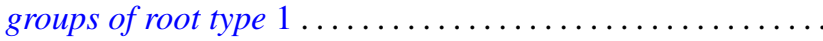

N. Stavrakas, A note on starshaped sets, $(k)$-extreme points and the half ray

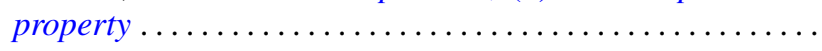

Carl E. Swenson, Direct sum subset decompositions of $Z \ldots \ldots$ 